\title{
Planck 2013 Cosmology Results: a Review
}

\author{
José Alberto Rubiño-Martín ${ }^{1,2}$ on behalf of the Planck Collaboration \\ ${ }^{1}$ Instituto de Astrofisica de Canarias, E-38200 La Laguna, Tenerife, Spain \\ ${ }^{2}$ Departamento de Astrofisica, Universidad de La Laguna, E-38206 La Laguna, Tenerife, Spain \\ Corresponding author: jalberto@iac.es
}

\begin{abstract}
This talk presents an overview of the cosmological results derived from the first 15.5 months of observations of the ESA's Planck mission. These cosmological results are mainly based on the Planck measurements of the cosmic microwave background (CMB) temperature and lensing-potential power spectra, although we also briefly discuss other aspects of the Planck data, as the statistical characterization of the reconstructed CMB maps, or the constraints on cosmological parameters using the number counts of galaxy clusters detected by means of the Sunyaev-Zeldovich effect in the Planck maps. All these results are described in detail in a series of papers released by ESA and the Planck collaboration in March 2013.
\end{abstract}

Keywords: cosmology: observations - cosmic microwave background - cosmological parameters - galaxy clusters.

\section{Introduction}

Planck $^{1}$ (Tauber et al., 2010; Planck Collaboration I, 2011 ) is the third generation space mission to measure the anisotropy of the cosmic microwave background $(\mathrm{CMB})$. It observes the sky in nine frequency bands covering 30-857 GHz with high sensitivity and angular resolution from 31' to 5'. The Low Frequency Instrument (LFI; Mandolesi et al., 2010; Bersanelli et al., 2010; Mennella et al., 2011) covers the 30, 44, and $70 \mathrm{GHz}$ bands with amplifiers cooled to $20 \mathrm{~K}$. The High Frequency Instrument (HFI; Lamarre et al., 2010; Planck HFI Core Team, 2011a) covers the 100, 143, 217, 353,545 , and $857 \mathrm{GHz}$ bands with bolometers cooled to $0.1 \mathrm{~K}$. Polarisation is measured in all but the highest two bands (Leahy et al., 2010; Rosset et al., 2010). A combination of radiative cooling and three mechanical coolers produces the temperatures needed for the detectors and optics (Planck Collaboration II, 2011). Two data processing centres (DPCs) check and calibrate the data and make maps of the sky (Planck HFI Core Team 2011b; Zacchei et al., 2011).

Planck's sensitivity, angular resolution, and frequency coverage make it a powerful instrument not only for cosmology, but also for Galactic and extra-galactic astrophysics. This talk focuses on the main cosmology results based on Planck's second data release, which covers data acquired in the period 12 August 2009 to 27 November 2010, and will be quoted here as nominal mission maps. All these results and the associated data products are described in a series of papers released in March 2013 (Planck Collaboration I-XXIX, 2013). Because the analysis of the polarization data is not yet as mature as the analysis of the temperature data, the Planck polarization results are not included in this release.

\section{Overview of Planck 2013 Cosmology Results}

The main goal of the Planck mission is to determine with great precision the key cosmological parameters describing our Universe, using the information encoded in the CMB anisotropies on intermediate and small angular scales over the whole sky (Tauber et al., 2010; Planck Collaboration I, 2013).

The data processing pipelines and beam calibrations employed by the LFI and HFI DPCs to create and characterize the nine full-sky maps based on the first 15.5 months of operations are described in Planck Collaboration II-IX (2013). These nine maps (see Table 1) allow robust reconstruction of the primordial CMB temperature fluctuations over nearly the full sky, as well as new constraints on Galactic foregrounds, including thermal dust and line emission from molecular carbon monoxide (Planck Collaboration XII 2013). Four different component separation methods were optimized to produce

1 Planck (http://www.esa.int/Planck) is a project of the European Space Agency (ESA) with instruments provided by two scientific consortia funded by ESA member states (in particular the lead countries France and Italy), with contributions from NASA (USA) and telescope reflectors provided by a collaboration between ESA and a scientific consortium led and funded by Denmark. 
a CMB map based on the Planck maps alone, i.e., without the addition of any other external data. Figure 1 shows the map produced by the SMICA method.

\subsection{The Planck CMB likelihood and the angular power spectrum}

A complete statistical description of the two-point correlation function of the CMB temperature fluctuations measured by Planck which accounts for all known relevant uncertainties, is encoded in the Planck likelihood (Planck Collaboration XV 2013). We follow a hybrid approach to construct this likelihood, using a exact approach at large scales $(\ell<50)$, and a pseudo- $\mathrm{C}_{\ell}$ approach at small scales $(\ell>50)$.

This function can be used to derive our estimate of the CMB angular power spectrum (see Figure 2), which constitutes an extremely precise measurement over three decades in multipole range, allowing to see seven acoustic peaks. In this plot, our main source of error at $\ell \leq 1500$ is due to cosmic variance. The measurements are in excellent agreement with previous experiments (WMAP, ACT, SPT).

We have validated our likelihood through an extensive list of consistency tests, assessing the impact of residual foreground and instrumental uncertainties on the final results. Although the polarization data are not released, we have checked that the best-fit $\Lambda \mathrm{CDM}$ cosmology is in excellent agreement with preliminary Planck EE and TE polarisation spectra.

Table 1: Planck performance parameters determined from flight data (Planck Collaboration I 2013).

\begin{tabular}{|l|c|c|c|}
\hline Channel & $\begin{array}{c}\nu_{\text {center }} \\
{[\mathrm{GHz}]}\end{array}$ & $\begin{array}{c}\text { FWHM } \\
{[\operatorname{arcmin}]}\end{array}$ & $\begin{array}{c}\text { Noise sensitivity } \\
{\left[\mu K_{\mathrm{RJ}} s^{1 / 2}\right]}\end{array}$ \\
\hline $30 \mathrm{GHz}$ & 28.4 & 33.16 & 145.4 \\
$40 \mathrm{GHz}$ & 44.1 & 28.09 & 164.8 \\
$70 \mathrm{GHz}$ & 70.4 & 13.08 & 133.9 \\
\hline $100 \mathrm{GHz}$ & 100 & 9.59 & 31.52 \\
$143 \mathrm{GHz}$ & 143 & 7.18 & 10.38 \\
$217 \mathrm{GHz}$ & 217 & 4.87 & 7.45 \\
$353 \mathrm{GHz}$ & 353 & 4.70 & 5.52 \\
$545 \mathrm{GHz}$ & 545 & 4.73 & 2.66 \\
$857 \mathrm{GHz}$ & 857 & 4.51 & 1.33 \\
\hline
\end{tabular}

\subsection{Lensing by large scale structure}

On small (arcminute) angular scales, the CMB anisotropies are perturbed by gravitational lensing, primarily sourced by the large scale structure of the Universe at relatively high redshifts (peaking at $z \sim 2$ ).
Planck provides a $25 \sigma$ detection of this effect (Planck Collaboration XVII, 2013), allowing also a lensing potential reconstruction at the map level on almost the full sky. The power spectrum of this lensing potential map is used to construct a "lensing" likelihood, which is included in our cosmological analyses.

The inclusion of the Planck lensing reconstruction can break degeneracies inherent in the temperature data alone, especially the geometric degeneracy in nonflat models, thus providing a strong constraint on spatial curvature without the need of including polarization data.

\subsection{Cosmological parameters: base model}

The Planck measurements of the CMB temperature and lensing-potential power spectra are well described by the standard spatially-flat six parameter $\Lambda \mathrm{CDM}$ model with adiabatic scalar perturbations (Planck Collaboration XVI, 2013). The computed values of the six key parameters within this model are summarized in Table 2, together with the values obtained for some derived parameters, as $\Omega_{\Lambda}, \Omega_{\mathrm{m}}, \sigma_{8}$ or the Hubble constant $H_{0}$.

The most remarkable result is that, for this cosmology, we find a low value of the Hubble constant, which is in tension with recent direct measurements of $H_{0}$, but is in excellent agreement with constraints from baryon acoustic oscillation surveys (see Planck Collaboration XVI (2013) for a detailed discussion). We also find a higher value of the matter density, as compared to previous measurements.

\subsection{Cosmological parameters: beyond the base model}

Multiple one-parameter extensions to the base (sixparameter) model have been discussed in Planck Collaboration XVI (2013). However, the main result is that there is no compelling evidence for any of these extensions.

For example, including curvature, we find that the Universe is consistent with spatial flatness to subpercent level precision $\left(\Omega_{\mathrm{tot}}=1.0010 \pm 0.0065\right.$, at $95 \%$, using Planck +lensing $+\mathrm{WP}+$ highL $+\mathrm{BAO})$. There is no evidence for additional neutrino-like relativistic particles beyond the three families of neutrinos in the standard model. Using $\mathrm{BAO}$ and $\mathrm{CMB}$ data, we find $N_{\text {eff }}=3.30 \pm 0.27$ for the effective number of relativistic degrees of freedom, in excellent agreement with the standard value of 3.046 , and an upper limit of $0.23 \mathrm{eV}$ for the sum of neutrino masses. In addition, we find no evidence for dynamical dark energy; using BAO and CMB data, the dark energy equation of state parameter 
is constrained to be $w=-1.13_{-0.10}^{+0.13}$.

Finally, deviations from the simplest inflationary scenario are discussed in Planck Collaboration XVI and XXII (2013), including tests of specific inflationary models, isocurvature modes, and broken scaleinvariance. As a main result, Planck rules out exact scale invariance $\left(n_{\mathrm{S}}=1\right)$ at high significance. The de- viation of the scalar spectral index from unity is insensitive to the addition of tensor modes and to changes in the matter content of the Universe. We find a $95 \%$ upper limit of $r<0.11$ on the tensor-to-scalar ratio (see Figure 3), which allows us to constrain a number of inflationary models.

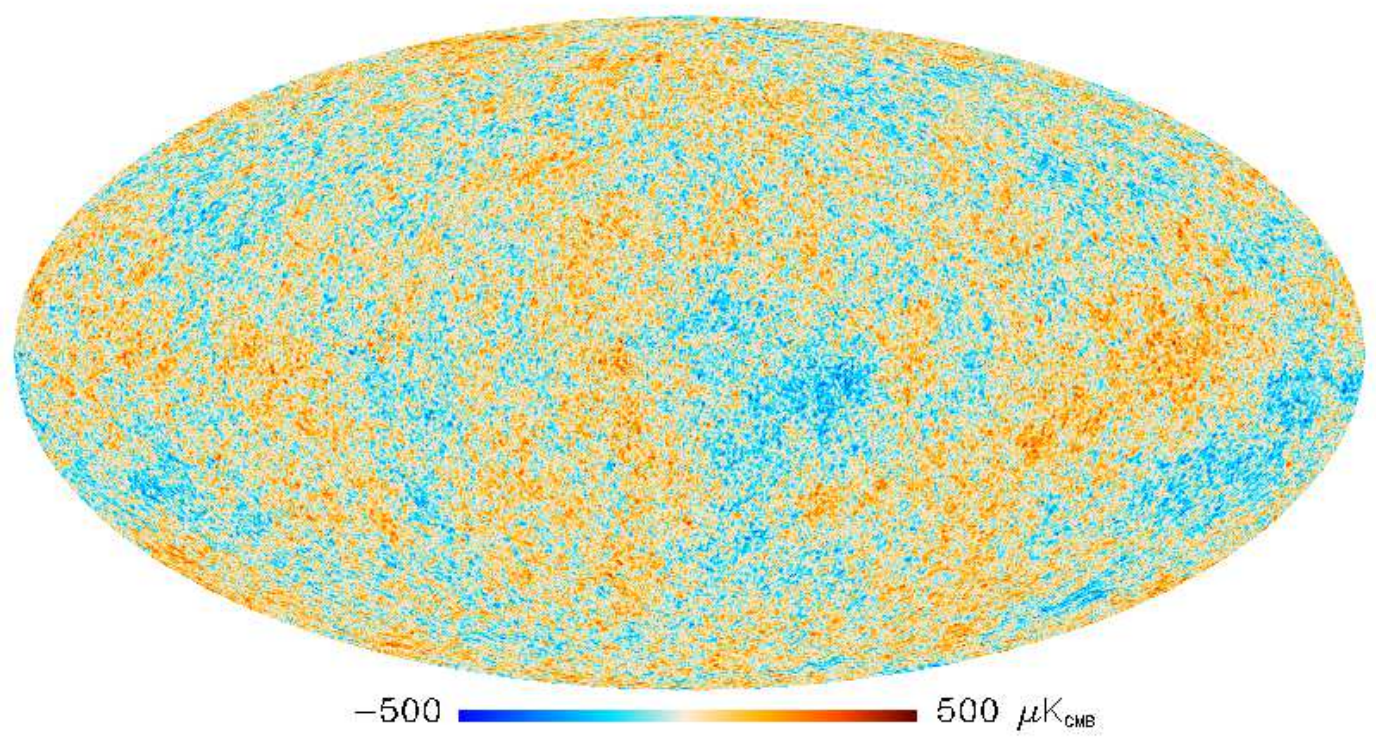

Figure 1: The SMICA method estimates the CMB over about $97 \%$ of the sky, with the remaining area replaced with a constrained Gaussian realization. Figure taken from Planck Collaboration I (2013).

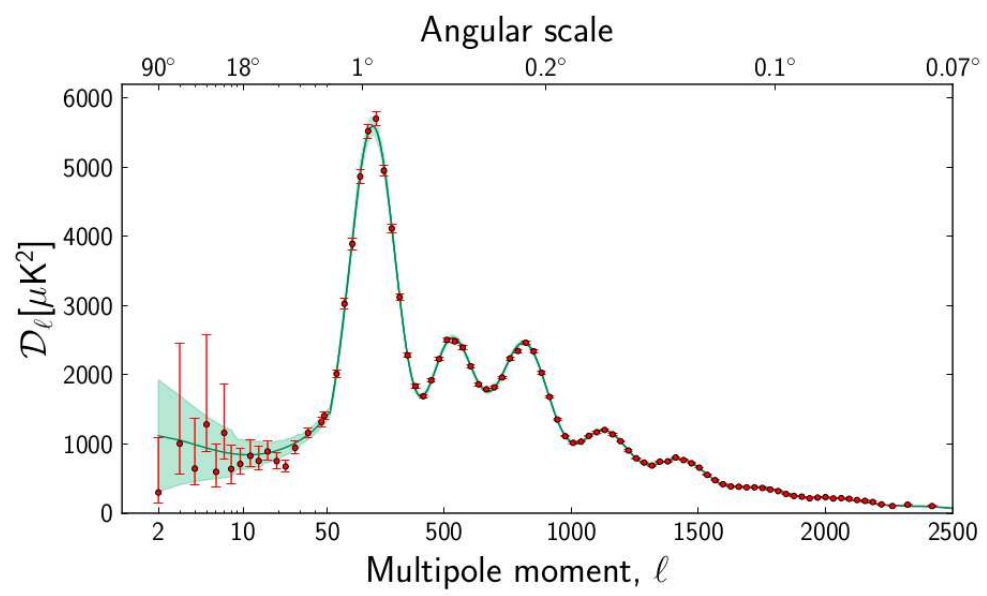

Figure 2: CMB temperature angular power spectrum from Planck. The shaded area represents cosmic variance. The solid line corresponds to the best-fit six-parameter $\Lambda \mathrm{CDM}$ model quoted in the text. Figure taken from Planck Collaboration I (2013). 
Table 2: Cosmological parameter values for the six-parameter base $\Lambda$ CDM model. Column 2 give the results for the Planck temperature and lensing potential power spectra, while column 3 adds the information from WMAP polarization (WP) at low multipoles $(\ell \leq 23)$, and other small scale CMB experiments (highL). See Planck Collaboration XVI 2013 for details. The last four rows are derived parameters.

\begin{tabular}{|l|c|c|}
\hline & Planck + lensing & + WP + highL \\
\hline$\Omega_{\mathrm{b}} h^{2}$ & $0.02217 \pm 0.00033$ & $0.02218 \pm 0.00026$ \\
$\Omega_{\mathrm{c}} h^{2}$ & $0.1186 \pm 0.0031$ & $0.1186 \pm 0.0022$ \\
$100 \theta_{\mathrm{MC}}$ & $1.04141 \pm 0.00067$ & $1.04144 \pm 0.00061$ \\
$\tau$ & $0.089 \pm 0.032$ & $0.090_{-0.014}^{+0.013}$ \\
$n_{\mathrm{S}}$ & $0.9635 \pm 0.0094$ & $0.9614 \pm 0.0063$ \\
$\ln \left(10^{10} A_{\mathrm{S}}\right)$ & $3.085 \pm 0.057$ & $3.087 \pm 0.024$ \\
\hline$\Omega_{\Lambda}$ & $0.693 \pm 0.019$ & $0.693 \pm 0.013$ \\
$\Omega_{\mathrm{m}}$ & $0.307 \pm 0.019$ & $0.307 \pm 0.013$ \\
$H_{0}$ & $67.9 \pm 1.5$ & $67.9 \pm 1.0$ \\
$\sigma_{8}$ & $0.823 \pm 0.018$ & $0.8233 \pm 0.0097$ \\
\hline
\end{tabular}
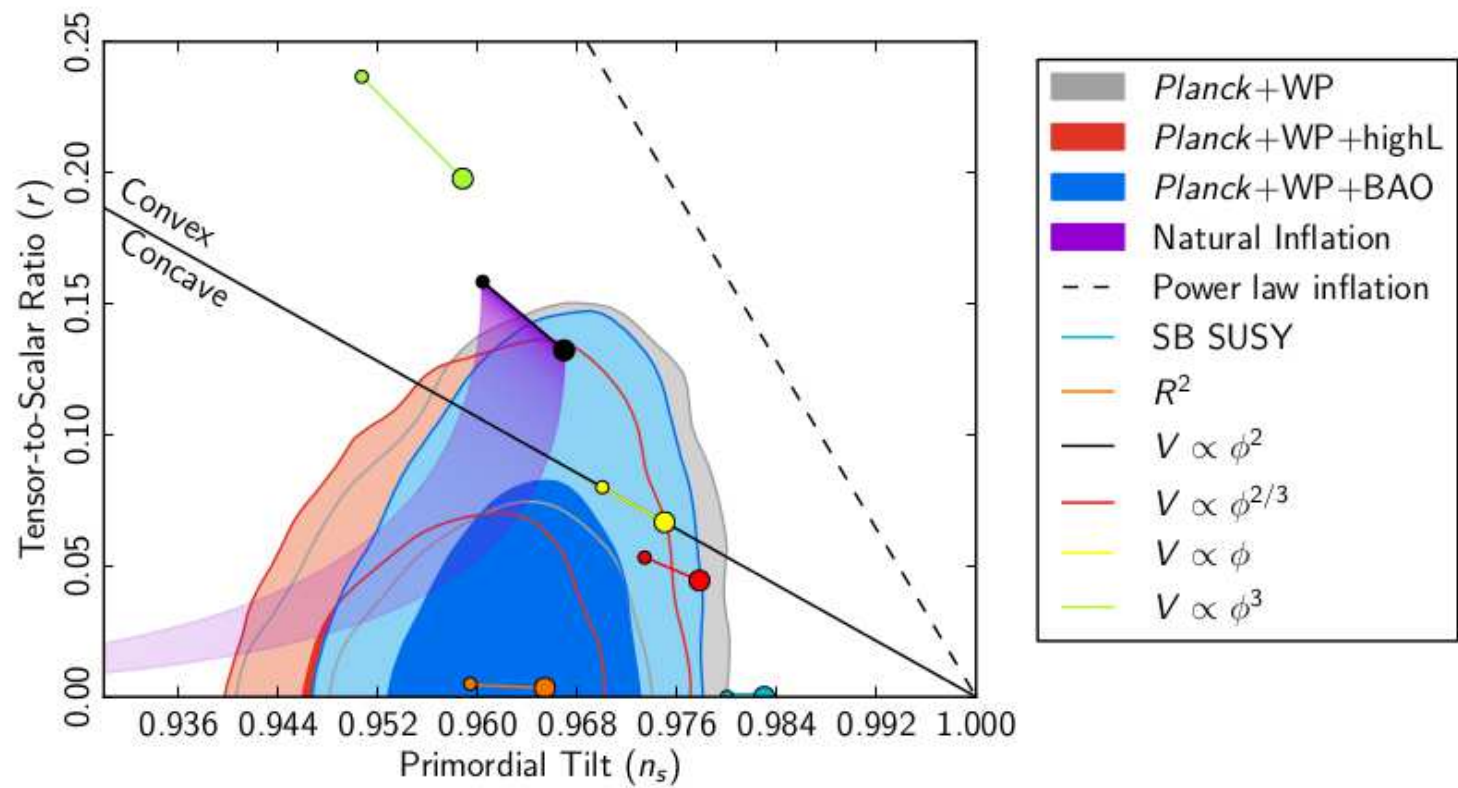

Figure 3: Marginalized joint $68 \%$ and $95 \%$ confidence level regions for $n_{\mathrm{S}}$ and $r$ from Planck in combination with other data sets, compared to the theoretical predictions of some inflationary models. Taken from Planck Collaboration XXII (2013).

\subsection{Gaussianity and statistical isotropy}

The statistical isotropy and the Gaussian nature of the Planck CMB maps has been rigorously examined in several papers (Planck Collaboration XXIII-XXVI, 2013). As a main result, there is no detection of primordial non Gaussianity, obtaining the following values for the primordial local, equilateral and orthogonal bispectrum amplitudes: $f_{\mathrm{NL}}^{\text {local }}=2.7 \pm 5.8, f_{\mathrm{NL}}^{\text {equil }}=-42 \pm 75$, and
$f_{\mathrm{NL}}^{\text {ortho }}=-25 \pm 39$ (all at $68 \%$ confidence level). The Integrated Sachs-Wolfe-lensing bispectrum signal is detected at 2-3 sigma level. The Planck data also provides stringent new constraints on cosmic strings and other defects (Planck Collaboration XXV 2013). Alternative geometries and non-trivial topologies have also been analyzed (Planck Collaboration XXVI 2013).

Deviations from isotropy have been found in Planck 
CMB maps, essentially confirming all the low-angle CMB anomalies previously seen in WMAP data at similar levels of significance (about $3 \sigma$ ): the low quadrupole amplitude; the quadrupole-octupole alignment; the hemispherical asymmetry; and the "cold spot". Finally, Planck notes a further low-angle discrepancy, related to the deficit of power in the angular power spectrum in the multipole range $20 \leq \ell \leq 40$, which could be connected with some of the previous features (e.g. the hemispherical asymmetry or the low observed variance).

\subsection{Galaxy clusters}

Planck maps can also be used to detect and study galaxy clusters by means of their Sunyaev-Zeldovich (SZ) effect (Sunyaev \& Zeldovich, 1972). Early Planck results on clusters were published in Planck Collaboration VIII-XII (2011). In March 2013, we have released the Planck catalogue of SZ sources (PSZ1) based on the nominal mission maps (Planck Collaboration XXIX 2013). PSZ1 contains 1227 entries, out of which 861 are confirmed galaxy clusters, and it constitutes the largest all-sky cluster catalogue to date (see Figure 4).

In Planck Collaboration XX (2013), we present constraints on cosmological parameters using the number counts $(N(z))$ for a well-defined and $100 \%$ reliable subsample of 189 galaxy clusters from PSZ1. Using a Xray calibrated mass-SZ flux relation, and assuming a $20 \%$ bias between the X-ray determined mass and the true mass of a cluster, we derive constraints on the matter power spectrum amplitude and the matter density parameter, obtaining $\sigma_{8}\left(\Omega_{\mathrm{m}} / 0.27\right)^{0.3}=0.78 \pm 0.01$, with one-dimensional ranges $\sigma_{8}=0.77 \pm 0.02$ and $\Omega_{\mathrm{m}}=0.29 \pm 0.02$.

These results are in excellent agreement with the cosmological constraints from the angular power spectrum of the thermal SZ emission. In Planck Collaboration XXI (2013), we construct the first all-sky maps of the thermal SZ emission, and compare their power spectrum to theoretical models, finding $\sigma_{8}\left(\Omega_{\mathrm{m}} / 0.27\right)^{0.3}=$ $0.784 \pm 0.016$.

However, these results favour somewhat low values of $\sigma_{8}$ and $\Omega_{\mathrm{m}}$ as compared to the CMB based analysis (see Table 2). This tension can be eliminated by relaxing the assumption on the mass bias between X-ray and true mass up to $45 \%$, although such a high value is difficult to reconcile with the known observed properties of galaxy clusters. There is another interesting possibility to reconcile these values, which consists in allowing for a component of massive neutrinos with a total mass (for the sum of the three species) of the order to $\sum m_{\nu} \approx 0.22 \mathrm{eV}$ (see Marulli et al. 2011 for a review of how cosmological observations can be affected by the inclusion of neutrino masses). These possibilities will be further investigated in future releases.

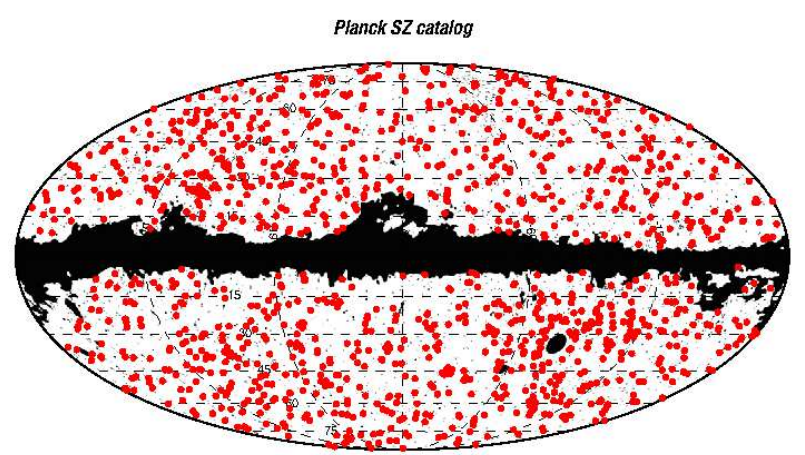

Figure 4: Distribution of the 1227 Planck SZ clusters and candidates across the sky, shown in Mollweide projection in Galactic coordinates. The masked regions in black color (point-sources, Magellanic clouds and Galactic emission) cover a total area of $16.3 \%$, and are not used by the SZ cluster-finder algorithms. Taken from Planck Collaboration XXIX (2013).

\section{Conclusions}

The main conclusions of the Planck 2013 release are:

- the Planck data are in remarkable agreement with a flat six-parameter $\Lambda \mathrm{CDM}$ model;

- we detect with high significance lensing of the CMB by intervening matter, providing evidence for dark energy from the CMB alone;

- the best-fit model contains a weaker cosmological constant $(\sim 69 \%)$, and more cold dark matter $(\sim 26 \%)$ than previously estimated; and we firmly establish a deviation from scale invariance for primordial matter perturbations;

- we find a low value of the Hubble constant, in tension with other astrophysical measurements;

- there is no compelling evidence for extensions of the base model;

- we find no evidence for significant deviations from Gaussianity in the statistics of CMB anisotropies; however, we confirm the anomalies at large angular scales first detected by $W M A P$ and find a deficit of power at low-multipoles with respect to our best-fit model.

- the Planck SZ cluster measurements are in tension with the CMB results; although this conclusion is limited by our knowledge of the observablemass relations, it might provide hints for physics in the neutrino sector. 
The next (2014) release of Planck data, which will include the full-mission maps and the polarization information, will provide a significant improvement in data quality and the level of systematic error control, helping in answering these open questions.

\section{Acknowledgement}

The development of Planck has been supported by: ESA; CNES and CNRS/INSU-IN2P3-INP (France); ASI, CNR, and INAF (Italy); NASA and DoE (USA); STFC and UKSA (UK); CSIC, MICINN, JA and RES (Spain); Tekes, AoF and CSC (Finland); DLR and MPG (Germany); CSA (Canada); DTU Space (Denmark); SER/SSO (Switzerland); RCN (Norway); SFI (Ireland); FCT/MCTES (Portugal); and PRACE (EU). A description of the Planck Collaboration and a list of its members, including the technical or scientific activities in which they have been involved, can be found at http://www.sciops.esa.int/index.php?project=planck. JAR-M is grateful to the organizers of the Frascati Workshop 2013 for the invitation to present these results. JAR-M acknowledges support from the project AYA2010-21766-C03-02 of the Spanish Ministry of Science and Innovation (MICINN).

\section{References}

[1] Bersanelli, M., Mandolesi, N., Butler, R. C., et al. 2010, A\&A, 520, A4

[2] Mandolesi, N., Bersanelli, M., Butler, R. C., et al. 2010, A\&A, 520, A3

[3] Mennella et al. 2011, A\&A, 536, A3

[4] Lamarre, J., Puget, J., Ade, P. A. R., et al. 2010, A\&A, 520, A9

[5] Leahy, J. P., Bersanelli, M., D'Arcangelo, O., et al. 2010, A\&A, 520, A8

[6] Marulli, F. et al. 2011, MNRAS, 418, 346

[7] Planck Collaboration I. 2011, A\&A, 536, A1

[8] Planck Collaboration II. 2011, A\&A, 536, A2

[9] Planck Collaboration VIII. 2011, A\&A, 536, A8

[10] Planck Collaboration IX. 2011, A\&A, 536, A9

[11] Planck Collaboration X. 2011, A\&A, 536, A10

[12] Planck Collaboration XI. 2011, A\&A, 536, A11

[13] Planck Collaboration XII. 2011, A\&A, 536, A12

[14] Planck HFI Core Team. 2011a, A\&A, 536, A4
[15] Planck HFI Core Team. 2011b, A\&A, 536, A6

[16] Planck Collaboration I. 2013, A\&A submitted

[17] Planck Collaboration II. 2013, A\&A submitted

[18] Planck Collaboration III. 2013, A\&A submitted

[19] Planck Collaboration IV. 2013, A\&A submitted

[20] Planck Collaboration V. 2013, A\&A submitted

[21] Planck Collaboration VI. 2013, A\&A submitted

[22] Planck Collaboration VII. 2013, A\&A submitted

[23] Planck Collaboration VIII. 2013, A\&A subm.

[24] Planck Collaboration IX. 2013, A\&A submitted

[25] Planck Collaboration X. 2013, A\&A submitted

[26] Planck Collaboration XII. 2013, A\&A submitted

[27] Planck Collaboration XIII. 2013, A\&A subm.

[28] Planck Collaboration XIV. 2013, A\&A subm.

[29] Planck Collaboration XV. 2013, A\&A submitted

[30] Planck Collaboration XVI. 2013, A\&A subm.

[31] Planck Collaboration XVII. 2013, A\&A subm.

[32] Planck Collaboration XVIII. 2013, A\&A subm.

[33] Planck Collaboration XIX. 2013, A\&A subm.

[34] Planck Collaboration XX. 2013, A\&A submitted

[35] Planck Collaboration XXI. 2013, A\&A subm.

[36] Planck Collaboration XXII. 2013, A\&A subm.

[37] Planck Collaboration XXIII. 2013, A\&A subm.

[38] Planck Collaboration XXIV. 2013, A\&A subm.

[39] Planck Collaboration XXV. 2013, A\&A subm.

[40] Planck Collaboration XXVI. 2013, A\&A subm.

[41] Planck Collaboration XXIX. 2013, A\&A subm.

[42] Rosset, C. et al. 2010, A\&A, 520, A13

[43] Sunyaev, R. A. \& Zeldovich, Y. B. 1972, Comments on Astrophysics and Space Physics, 4, 173

[44] Tauber, J. A. et al. 2010, A\&A, 520, A1

[45] Zacchei et al. 2011, A\&A, 536, A5 


\section{DISCUSSION}

MARCO REGIS: Can the large scale anomalies be due to galactic foregrounds?

JOSE ALBERTO RUBIÑO-MARTIN: We have performed a large number of tests using the four $\mathrm{CMB}$ maps produced by the different component separation algorithms, and the consistency of the results strongly favors a cosmological origin for the anomalies. In addition, the agreement between Planck and WMAP rules out a possible explanation based on systematic effects. 